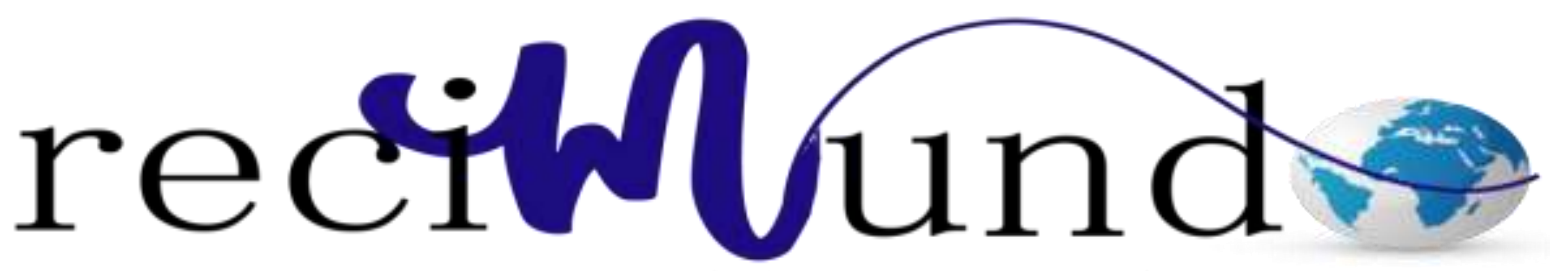

Revista Cientifica Mundo de la Investigación y el Conocimiento

\author{
María Monserrate Ganchozo Pincay ${ }^{\text {a }}$; Charles David Altamirano Olvera ${ }^{\text {b; Renato }}$ \\ Paolo Patiño Andrade c ${ }^{c}$ Javier Alexander Beltrán Bayas d
}

Determinar las complicaciones de las fracturas expuestas de tibia en pacientes de 20-40 años en el hospital Teodoro Maldonado Carbo periodo 2014

To determine the complications of tibia exposed fractures in patients aged 20-40 years in the Teodoro Maldonado Carbo hospital 2014 period

Revista Científica Mundo de la Investigación y el Conocimiento. Vol. 3 núm.3, septiembre, ISSN: 2588-073X, 2019, pp. 807-823

DOI: $\underline{10.26820 / \text { recimundo/3.(3).septiembre.2019.807-823 }}$

\begin{abstract}
URL: http://recimundo.com/index.php/es/article/view/550
\end{abstract}
Código UNESCO: 3205 Medicina Interna

Tipo de Investigación: Artículo de Revisión

Editorial Saberes del Conocimiento

Recibido: 15/05/2019

Aceptado: 23/06/2019

Publicado: 30/09/2019

Correspondencia: gp_mayte@ hotmail.com
a. Médico; Investigador Independiente; Guayaquil, Ecuador; gp_mayte@ hotmail.com
b. Médico; Investigador Independiente; Guayaquil, Ecuador; chardei27_29@ hotmail.com
c. Médico; Investigador Independiente; Guayaquil, Ecuador; polo_rp69@hotmail.com
d. Médico; Investigador Independiente; Guayaquil, Ecuador; javicob85@gmail.com 


\section{Determinar las complicaciones de las fracturas expuestas de tibia en pacientes de 20-40 años en el hospital Teodoro Maldonado Carbo periodo 2014}

Vol. 3, núm. 3., (2019)

Maria Monserrate Ganchozo Pincay; Charles David Altamirano Olvera; Renato Paolo Patiño; Andrade; Javier Alexander Beltrán Bayas

\section{RESUMEN}

Las fracturas expuestas de tibia han constituido durante mucho tiempo un gran problema de salud pública. Tiene en común tres factores de riesgo fundamentales: alta posibilidad de contaminación, gran daño de tejidos blandos y hueso, así como dificultades para el manejo conservador y quirúrgico a causa del daño óseo y de partes blandas asociados que establece un alto índice de complicaciones. El tratamiento de estas fracturas es un tema aún controvertido especialmente en la modalidad quirúrgica a utilizar. La precaria vascularización y poca cantidad de tejidos blandos que cuben la tibia convierten a este hueso muy vulnerable a la pseudoartrosis, la infección y a otras complicaciones. En la actualidad, se dispone de una gran variedad de tratamientos para reducir las complicaciones señaladas. Se realiza un estudio observacional descriptivo de 118 pacientes con Diagnóstico de Fracturas Expuestas de Tibia en pacientes de 20 a 40 años captados desde 1 de enero de 2014 al 31 de diciembre del 2014.

Palabra Clave: Complicación; Tibia; Partes blandas; Infección; Fractura. 


\title{
Determinar las complicaciones de las fracturas expuestas de tibia en pacientes de 20-40 años en el hospital Teodoro Maldonado Carbo periodo 2014
}

Vol. 3, núm. 3., (2019)

Maria Monserrate Ganchozo Pincay; Charles David Altamirano Olvera; Renato Paolo Patiño; Andrade; Javier Alexander Beltrán Bayas

\begin{abstract}
The open tibial fractures has long constituted a major public health problem. It has in common three key risk factors: high posibility of contamination, large soft tissue damage and bone, as well as difficulties for the conservative and surgical management because of bone and soft tissue damage associated with a high complication rate. The treatment of these fractures is still controversial topic especially in the surgical modality used. The precarious vascularization and a small amount of soft tissue covering the tibia make this very vulnerable to bone nonunion, infection and another complications. At present, there is a variety of treatments to reduce the aforementioned complications. A descriptive study of 118 patients diagnosed in patiens 20 to 40 years recruited from January 1, 2014 to December 31, 2014 is made.
\end{abstract}

Key words: Complication; Warm; Soft tissue infection; Fracture. 


\section{Determinar las complicaciones de las fracturas expuestas de tibia en pacientes de 20-40 años en el hospital Teodoro Maldonado Carbo periodo 2014}

Vol. 3, núm. 3., (2019)

Maria Monserrate Ganchozo Pincay; Charles David Altamirano Olvera; Renato Paolo Patiño; Andrade; Javier Alexander Beltrán Bayas

\section{Introducción.}

Las fracturas expuestas de tibia constituyen un fuerte problema de Salud frecuente en adolescentes y en adultos, además originan elevados costos de tratamiento. Este tipo de fracturas son en la mayoría de los casos secundarias accidentes de tránsito constituyen.

La tibia es un hueso largo, par y asimétrico; situado en la parte anterior e interna de la pierna; por dentro del peroné con el cual se articula por sus extremos, por debajo del fémur, $\mathrm{q}$ descansa sobre ella, y por encima del conglomerado óseo del tarso, el cual en transmisión vertical transmite el peso del cuerpo. De los huesos de la pierna es con mucho el más voluminoso y el más importante. (Testud \& A., 1979)

Las fracturas son soluciones de continuidad que se originan en los huesos a consecuencias de golpes, fuerzas, tracciones cuyas intensidades superan la elasticidad del hueso, como consecuencia de la aplicación de una fuerza sobre el esqueleto, de forma directa o indirecta, se puede producir una lesión osteoarticular. Un traumatismo en las extremidades pocas veces origina una situación de riesgo vital, pero puede ocasionar discapacidades. En cualquier caso, de traumatismo es importante conocer el antecedente que ha originado la lesión. (Muller, Allgowe, \& Schneider, 1994)

Ecuador es el cuarto país en el mundo y el segundo en América Latina, en donde los accidentes de tránsito se convierten en la principal causa de muerte, dejando como principal consecuencia la presencia de fracturas que dependiendo de magnitud podría presentar varias 


\section{Determinar las complicaciones de las fracturas expuestas de tibia en pacientes de 20-40 años en el hospital Teodoro Maldonado Carbo periodo 2014}

Vol. 3, núm. 3., (2019)

Maria Monserrate Ganchozo Pincay; Charles David Altamirano Olvera; Renato Paolo Patiño; Andrade; Javier Alexander Beltrán Bayas

complicaciones que pueden llegar a la muerte. En el 2009 en el Ecuador se registraron 4.693 accidentes de tránsito a nivel nacional, excepto de la provincia del Guayas, información que se concentra en la Comisión de Tránsito del Guayas (CTG). La clasificación tipos de accidentes por el SIAT a nivel de país, está relacionado con choque lateral, estrellamiento, choque lateral perpendicular, choque por alcance, y atropellamiento entre los más importantes. La ingesta de bebidas alcohólicas se establece como la causa más importante para la accidentabilidad, alcanzando el 60,98\% de las causas concurrente de accidentes de tránsito. En lo que respecta al tipo de automotores involucrados en los accidentes de tránsito, las motocicletas se ubican en el tercer puesto con el 11,33\%. (Fuente: Siat Anuario Estadístico 2009 - *Excepto información de la provincia del Guayas - Elaboración: Secretaría de Salud).

Un elemento importante en el manejo de enfermos con FAT es el hecho que más de la mitad de los afectados presentan algún tipo de lesión asociada, es esta la razón por la que estos pacientes deben ser considerados dentro del grupo de politraumatizados y desde su inicio realizar una valoración integral del enfermo, empezando por los aspectos más vitales.

En los últimos años un aumento de la incidencia de accidentes de tránsito ha llevado incluso a duplicar la incidencia de fracturas expuestas de tibia dentro de las fracturas más frecuentes de miembros inferiores y fracturas a nivel general. Se estima que alrededor del 70\% de la población adulta y adolescente sufre un tipo de accidente de tránsito al año y de estos un $30 \%$ presentan fracturas expuestas de tibia. 


\section{Determinar las complicaciones de las fracturas expuestas de tibia en pacientes de 20-40 años en el hospital Teodoro Maldonado Carbo periodo 2014}

Vol. 3, núm. 3., (2019)

Maria Monserrate Ganchozo Pincay; Charles David Altamirano Olvera; Renato Paolo Patiño; Andrade; Javier Alexander Beltrán Bayas

El impacto de las fracturas de expuestas de tibia muchas veces es catastrófico, relacionado con deterioro marcado de su movilidad y estado funcional, es fundamental un manejo médico inicial inmediato dentro de las primeras 6 horas posterior al trauma ; así como la clasificación respectiva y el consecuente control de la evolución del paciente hospitalizado, permitiendo mejorar el curso clínico más la posibilidad de un tratamiento quirúrgico, disminuyendo la estancia y mejorar el pronóstico rehabilitador.

La hospitalización del paciente con fracturas expuestas de tibia es de riesgo para una serie de complicaciones desde leves hasta graves. Lesiones vasculares, lesiones de nervios periféricos, lesiones viscerales, shock traumático, embolia grasa, síndrome compartimental, osteomielitis, miositis osificante y tétanos.

Actualmente los recursos sistémicos han permitido conocer mejores evidencias respecto al manejo perioperatorio de estos pacientes, así son aceptadas como beneficiosa la antibioticoterapia profiláctica y la anticoagulación profiláctica.

El propósito de la investigación es determinar las complicaciones de las Fracturas Expuestas de Tibia según la Clasificación de Gustillo en paciente entre los 20 a 40 años de edad y elaborar una guía de manejo, para de esta manera transferir y actualizar información de esta patología de gran demanda en nuestro hospital y así obtener un índice menor de complicaciones. Se analizó los antecedentes evolutivos de la enfermedad, su incidencia a nivel mundial, epidemiología, historia natural, manifestaciones clínicas, métodos complementarios de diagnóstico y tratamientos vigentes hasta la actualidad. 


\section{Determinar las complicaciones de las fracturas expuestas de tibia en pacientes de 20-40 años en el hospital Teodoro Maldonado Carbo periodo 2014}

Vol. 3, núm. 3., (2019)

Maria Monserrate Ganchozo Pincay; Charles David Altamirano Olvera; Renato Paolo Patiño; Andrade; Javier Alexander Beltrán Bayas

\section{Metodología}

Es un estudio observacional, descriptivo, de corte longitudinal y prospectivo. Se analizó todos los pacientes que ingresaron con Diagnóstico de Fracturas Expuestas de Tibia en el Servicio de Emergencia, Hospitalización y Consulta Externa de Traumatología y Ortopedia del Hospital IESS Dr. Teodoro Maldonado Carbo en el período comprendido entre el 1 de Enero del 2014 hasta el 31 de Diciembre del 2014. Se analizó el índice de pacientes que acudieron a la Emergencia del Servicio de Traumatología y Ortopedia del Hospital IESS Dr. Teodoro Maldonado Carbo con Diagnóstico de Fracturas Expuestas de Tibia y su relación con tiempo de consulta desde el evento traumático, tiempo de espera del tratamiento, tiempo de estancia hospitalaria, tipo de lesión, complicaciones, control posterior y resultados generales.

La evaluación se llevó a cabo a través de un estudio estadístico y prospectivo del examen de los pacientes, del análisis de las historias clínicas de los mismos con sus respectivos estudios complementarios y terapéuticas implementadas en el transcurso de la evaluación. Además del control posterior a su tratamiento y la evidencia d complicaciones; se registró en una ficha de datos desarrollada por el investigador.

Se analizó la información obtenida en el Software Excel y Word, esta presentada en forma de tablas, gráficos para una mejor comprensión, de acuerdo a las variables de estudio. Se utilizó estadística descriptiva e interferencial para el análisis de los datos y se utilizaron pruebas no paramétricas para medir las relaciones variables cualitativas. 


\section{Determinar las complicaciones de las fracturas expuestas de tibia en pacientes de 20-40 años en el hospital Teodoro Maldonado Carbo periodo 2014}

Vol. 3, núm. 3., (2019)

Maria Monserrate Ganchozo Pincay; Charles David Altamirano Olvera; Renato Paolo Patiño; Andrade; Javier Alexander Beltrán Bayas

Para una mejor recolección de datos y comprensión de la información se realizó una ficha de datos, en la que se tomaron a consideración las variables antes descritas, y parámetros específicos para información selectiva y detallada.

\section{Resultados}

En función de los objetivos y de la hipótesis se procede al análisis e interpretación de resultados.

Tabla 1. Comportamiento de indicadores de edad y sexo en que presentan las Fracturas Expuestas de Tibia.

\begin{tabular}{|l|l|l|}
\hline Sexo & Número & Porcentaje \\
\hline Masculino & 99 & $84 \%$ \\
\hline Femenino & 19 & $16 \%$ \\
\hline TOTAL & 118 & $100 \%$ \\
\hline
\end{tabular}

Fuente: Historia Clínica - HRTMC

Autor: Investigador

El orden de frecuencia de este tipo de lesiones, presentándose en el $84 \%$ de los casos en el sexo masculino y el $16 \%$ en el sexo femenino. 
Determinar las complicaciones de las fracturas expuestas de tibia en pacientes de 20-40 años en el hospital Teodoro Maldonado Carbo periodo 2014

Vol. 3, núm. 3., (2019)

Maria Monserrate Ganchozo Pincay; Charles David Altamirano Olvera; Renato Paolo Patiño; Andrade; Javier Alexander Beltrán Bayas

\section{Tabla. 2. Distribución según la Etiología}

\begin{tabular}{|l|l|l|}
\hline Etiología & Número & Porcentaje \\
\hline Accidente de tránsito & 90 & $76 \%$ \\
\hline Accidente laboral & 21 & $17 \%$ \\
\hline Caída de altura & 7 & $6 \%$ \\
\hline TOTAL & $\mathbf{1 1 8}$ & $\mathbf{1 0 0 \%}$ \\
\hline
\end{tabular}

Fuente: Historia Clínica - HRTMC

Autor: Investigador

La causa más frecuente de Fracturas Expuestas de Tibia en nuestro estudio fueron los accidentes de tránsito con 90 casos (76\%), seguido de accidentes laborales con 21 casos (18\%) y caída de altura con 7 casos (6\%).

Tabla 3. Número de pacientes según el grado de exposición de acuerdo a la Clasificación de Gustilo y Anderson

\begin{tabular}{|l|l|l|}
\hline Grado de Exposición & Número & Porcentaje \\
\hline Grado I & 12 & $10 \%$ \\
\hline Grado II & 46 & $39 \%$ \\
\hline Grado III A & 52 & $44 \%$ \\
\hline Grado III B & 8 & $7 \%$ \\
\hline TOTAL & $\mathbf{1 1 8}$ & $\mathbf{1 0 0 \%}$ \\
\hline
\end{tabular}

Fuente: Historias Clínicas - HRTMC

Autor: Investigador

Según los pacientes estudiados que predominó el Tipo IIIA de Gustilo con 52 casos (44\%), seguido por el tipo II con 46 casos (39\%), en orden decreciente se encontró que el Tipo I con 12 casos (10\%) y el Tipo III B con 8 casos (7\%). 
Determinar las complicaciones de las fracturas expuestas de tibia en pacientes de 20-40 años en el hospital Teodoro Maldonado Carbo periodo 2014

Vol. 3, núm. 3., (2019)

Maria Monserrate Ganchozo Pincay; Charles David Altamirano Olvera; Renato Paolo Patiño; Andrade; Javier Alexander Beltrán Bayas

\section{Tabla 4. Distribución según el mecanismo lesional}

\begin{tabular}{|l|l|l|}
\hline Mecanismo Lesional & Número & Porcentaje \\
\hline Directo & 100 & $85 \%$ \\
\hline Indirecto & 18 & $15 \%$ \\
\hline TOTAL & $\mathbf{1 1 8}$ & $\mathbf{1 0 0 \%}$ \\
\hline
\end{tabular}

Fuente: Historias Clínicas - HRTMC

Autor: Investigador

El mecanismo lesional más frecuente en nuestro estudio fue el traumatismo directo con 100 casos $(85 \%)$ seguido por los traumatismos indirectos con 18 casos $(15 \%)$.

Tabla 5. Modalidad de tratamiento utilizado

\begin{tabular}{|l|l|l|}
\hline Tratamiento & Número & Porcentaje \\
\hline Tracción esquelética y yeso & 9 & $8 \%$ \\
\hline Fijación externa & 62 & $53 \%$ \\
\hline Intramedulares & 31 & $26 \%$ \\
\hline Placas AO & 16 & $13 \%$ \\
\hline TOTAL & $\mathbf{1 1 8}$ & $\mathbf{1 0 0 \%}$ \\
\hline
\end{tabular}

Fuente: Historias Clínicas - HRTMC

Autor: Investigador

El Fijador Externo fue la modalidad de tratamiento más utilizada con 62 casos (53\%), en orden decreciente se encontró fijación intramedular con 31 casos (26\%), seguido por placas AO con 16 casos (13\%) y tracción esquelética y yeso en 9 casos $(8 \%)$.

Tabla 6. Número de pacientes según las complicaciones encontradas

\begin{tabular}{|l|l|l|}
\hline Complicaciones & Número & Porcentajes \\
\hline SI & 47 & $40 \%$ \\
\hline NO & 71 & $60 \%$ \\
\hline TOTAL & 118 & $100 \%$ \\
\hline
\end{tabular}

Fuente: Historias Clínicas - HRTMC

Autor: Investigador 


\section{Determinar las complicaciones de las fracturas expuestas de tibia en pacientes de 20-40 años en el hospital Teodoro Maldonado Carbo periodo 2014}

Vol. 3, núm. 3., (2019)

Maria Monserrate Ganchozo Pincay; Charles David Altamirano Olvera; Renato Paolo Patiño; Andrade; Javier Alexander Beltrán Bayas

Hubo 47 casos (40\%) que, si presentaron complicaciones, frente a 71 casos (60\%) que no las presentaron durante el período de estudio.

Tabla 7. Comportamiento según complicaciones

\begin{tabular}{|l|l|l|}
\hline Complicaciones & Número & Porcentajes \\
\hline Pseudoartrosis & 6 & $5 \%$ \\
\hline Retardo de consolidación & 7 & $6 \%$ \\
\hline Infección & 19 & $16 \%$ \\
\hline Acortamiento & 5 & $4 \%$ \\
\hline Amputación & 4 & $3 \%$ \\
\hline Síndrome Compartimental & 6 & $5 \%$ \\
\hline TOTAL & $\mathbf{4 7}$ & $\mathbf{4 0 \%}$ \\
\hline
\end{tabular}

Fuente: Historia Clínica - HRTMC

Autor: Investigador

Se observa la siguiente distribución de las complicaciones:

- Pseudoartrosis: $5 \%$

- Retardo de consolidación: $6 \%$

- Infección: $16 \%$

- Acortamiento: $4 \%$

- Amputación: 3\%

- Síndrome Compartimental: $5 \%$

Las fracturas abiertas de tibia predominan en el sexo masculino según los resultados que plantea Álvarez y Cassanova et al, no difieren de nuestra investigación ya que se presentó 99 casos (84\%) en varones y 19 casos (16\%). La mayoría de los autores informan que este tipo de fracturas 


\section{Determinar las complicaciones de las fracturas expuestas de tibia en pacientes de 20-40 años en el hospital Teodoro Maldonado Carbo periodo 2014}

Vol. 3, núm. 3., (2019)

Maria Monserrate Ganchozo Pincay; Charles David Altamirano Olvera; Renato Paolo Patiño; Andrade; Javier Alexander Beltrán Bayas

es predominante en la tercera y cuarta etapa de la vida ya que están más expuestas accidentes, dato muy similar fue obtenido en este trabajo, recordando que se asoció y se tomó en cuenta como parte del título de nuestro tema de investigación por la frecuencia citada por muchos autores. Los accidentes de tránsito, específicamente son los de mayor incidencia en ésta afección según lo obtenido por el este estudio, con 36 casos (59\%), seguido por accidente de trabajo $24,5 \%$ y otros; en un estudio tipo descriptivo y transversal en 61 pacientes tratados con fracturas abiertas de tibia en el Hospital Provincial Docente Clínico Quirúrgico “Manuel Ascunce Domenech”. Para originarse una fractura abierta se necesita un mecanismo de alta energía, las fracturas abiertas de tibia tipo II de la Clasificación de Gustillo y Anderson son las más comunes con 33 casos (54\%) en el estudio citado por Álvarez y Casanova et al, que asocian de igual manera como complicación principal es el retardo de consolidación en 12 casos $(19,6 \%)$ seguido de infección con 10 casos $(16,3 \%$.) y muy diferente a lo obtenido por nuestro estudio que indica que el Grado de Fractura con mayor frecuencia se asocia a la fracturas tipo IIIA de la Clasificación de Gustillo y Anderson con 52 casos (44\%), seguido por las fracturas tipo II de la clasificación antes citada con 46 casos (39\%) y la tipo I en orden decreciente con 12 casos (10\%).

En nuestro estudio del $100 \%$ solo el $40 \%$ presentaron complicaciones siendo la más frecuente los procesos infecciosos con 19 casos (16\%), en orden decreciente el retardo de consolidación con 7 casos $(6 \%)$ y síndrome compartimental y pseudoartrosis en un mismo porcentaje (5\%). Reconociendo de esta manera que las complicaciones que se presentan no varían mucho de las citadas por Álvarez y Casanova et al, que tomaron como determinantes variables similares a las de nuestro estudio de campo. 


\section{Determinar las complicaciones de las fracturas expuestas de tibia en pacientes de 20-40 años en el hospital Teodoro Maldonado Carbo periodo 2014}

Vol. 3, núm. 3., (2019)

Maria Monserrate Ganchozo Pincay; Charles David Altamirano Olvera; Renato Paolo Patiño; Andrade; Javier Alexander Beltrán Bayas

Todas las fracturas abiertas deben considerarse contaminadas debido a la comunicación existente entre el foco de fractura y el ambiente exterior. Charalampos y Patzakis et al, asocian que el riesgo de infección oscila entre el 0 y el 2\% para el tipo I, el 2 al 10\% al tipo II y el 50\% al tipo III, rangos similares se observaron en nuestro estudio. El papel esencial de la administración de antibióticos se demostró en este estudio prospectivo y aleatorizado quienes demostraron una reducción marcada en la incidencia de la infección al administrar Cefazolina 2,8\% (8/84) en comparación con la no utilización de antibióticos 13,9\% (11/79). Aunque en un contexto en general nuestro estudio de investigación tomo en consideración el tiempo transcurrido desde accidente hasta la primera atención y cobertura antibiótica en las que se observó que pacientes fueron atendidos en unos 65 casos (55\%) mayor a las 6 primeras horas luego del accidente con cobertura antibiótica de inicio y posterior tratamiento quirúrgico en los cuales sobresalió un tiempo mayor a las $12 \mathrm{~h}$ en 51 casos $(43 \%)$. Se tomó tales medidas y variantes como principales puntos de discusión de sobre cuál era el momento ideal para el comienzo del tratamiento, la duración del tratamiento antibiótico y la mejor combinación de fármacos.

Aún y más allá de inicio de esquema terapéutico y las medidas de tratamiento en sí, se tomó a consideración en nuestra investigación de gran determinante la lesión de partes blandas que con mucha frecuencia es subestimada en la primera valoración del paciente con lo subsiguiente que esto provoca; para conseguir resultados aceptables es imprescindible un tratamiento protocolizado e integrado tanto en lesiones ósea y partes blandas. Muñóz y Doussoux et al, en un estudio transversal, aleatorio, con 21 pacientes demostraron que el desbridamiento quirúrgico inicial y un tanto agresivo fueron satisfactorios de los cuales 15 casos (71\%) y con 6 casos (29\%) sin cambios 


\section{Determinar las complicaciones de las fracturas expuestas de tibia en pacientes de 20-40 años en el hospital Teodoro Maldonado Carbo periodo 2014}

Vol. 3, núm. 3., (2019)

Maria Monserrate Ganchozo Pincay; Charles David Altamirano Olvera; Renato Paolo Patiño; Andrade; Javier Alexander Beltrán Bayas

significativos, demostraron mejores resultados funcionales y de consolidación de las propias fracturas. Nuestro estudio difiere un poco de los resultados obtenidos en el estudio citado observándose cambios pocos significativos en el desbridamiento precoz y agresivo con 9 casos (8\%) frente al desbridamiento consecutivo 51 casos $(43,2 \%)$.

Para Tornetta y cols, tanto el tratamiento con el enclavado intramedular no fresado como la fijación externa se han empleado ampliamente en el tratamiento de las fracturas abiertas de tibia, en su estudio prospectivo y aleatorio estudiaron los métodos en 29 fracturas abiertas de tibia tipo, todas las fracturas consolidaron y no se encontraron diferencias, en nuestro investigación hay notable diferencia de resultados el tratamiento q se utilizó con mayor frecuencia aun tomando en consideración el grado de lesión fue de 62 casos (53\%) para la fijación externa, seguido de 31 casos $(21 \%)$ con los intramedulares, sin notables diferencias.

\section{Conclusiones}

Las Fracturas Expuestas de tibia es una lesión que representa un grave problema de la salud pública, ha aumentado su frecuencia en los últimos años en el Hospital Regional IESS Teodoro Maldonado Carbo, en el tiempo que transcurrió el estudio, debido al aumento de afiliados que consulta a la institución.

En la actualidad representa en nuestro país y en el mundo una de las lesiones más frecuentes a nivel de miembros inferiores, causadas por los accidentes de tránsito ocasionando un alto índice de mortalidad, pérdidas económicas, secuelas psicológicas y físicas. 


\section{Determinar las complicaciones de las fracturas expuestas de tibia en pacientes de 20-40 años en el hospital Teodoro Maldonado Carbo periodo 2014}

Vol. 3, núm. 3., (2019)

Maria Monserrate Ganchozo Pincay; Charles David Altamirano Olvera; Renato Paolo Patiño; Andrade; Javier Alexander Beltrán Bayas

La población más afectada fue el sexo masculino con el 99 caso (84\%), con un rango entre

20 a 37 años con actividad física y deportiva, se asocia a población joven debido a la falta de concientización, abuso de la velocidad y el consumo excesivo de alcohol y acompañados de un medio de transporte en la actualidad de bajo coste, fácil manejo y acceso como lo son las motocicletas.

Al hablar de fracturas expuestas de tibia para que se produzca está lesión se necesita un mecanismo de alta energía. La causa más frecuente de Fracturas Expuestas de tibia en nuestro estudio, fueron los accidentes de tránsito con 90 casos (76\%), se produjo en su mayoría en forma directa con 100 casos (85\%), lo que indica que los traumatismos de alta energía superan el límite de resistencia del hueso. La localización más frecuente fue la diáfisis de la tibia con el (53\%) de los casos; y esto se debe a su localización anatómica en miembros inferiores y situación subcutánea con pobre cobertura de partes blandas.

El tipo de exposición se basó en la Clasificación de Gustilo y Anderson observándose en nuestro estudio con mayor frecuencia la Tipo IIIA con 52 casos (44\%) y seguido por el tipo II con 46 casos (39\%). La estabilización de las fracturas expuestas de tibia fue la fijación interna que es el gold standard con 62 casos (53\%); aunque en la Grado I se utilizó con mayor frecuencia la osteosíntesis y en el grado IIIC se realizó la amputación de la extremidad afectada en la mayoría de los casos; y todo esto debido a que el riesgo de infección está relacionado con la gravedad de la lesión. 


\section{Determinar las complicaciones de las fracturas expuestas de tibia en pacientes de 20-40 años en el hospital Teodoro Maldonado Carbo periodo 2014}

Vol. 3, núm. 3., (2019)

Maria Monserrate Ganchozo Pincay; Charles David Altamirano Olvera; Renato Paolo Patiño; Andrade; Javier Alexander Beltrán Bayas

El número de limpiezas quirúrgicas y desbridamiento que se realizó a los pacientes antes de tratamiento definitivo estuvo en relación directa con el grado de lesión de partes blandas y de las complicaciones. Este tipo de lesiones cursa con un grado importante de complicaciones por lo que nuestro estudio registro de los 118 pacientes 47 casos (40\%), siendo los procesos infecciosos la complicación más frecuente con 19 casos (16\%) y cuyo patógeno aislado fue la Pseudomona aureginosa con (11 cultivos positivos).

La estancia hospitalaria fue mayor en las fracturas IIIA y IIIB de 30 a 90 días (63\% - 36\% respectivamente) siendo directamente proporcional a las lesiones asociadas y complicaciones. Además del tiempo transcurrido desde el accidente hasta la primera intervención quirúrgica que suele superar las $6 \mathrm{~h}$ en $(75 \%)$ de los casos y que es el tiempo de oro de una fractura expuesta.

Sobre la base de nuestros datos concluimos que existen determinantes específicas que nos ayudan a valorar, diagnosticar y tratar las fracturas expuestas de tibia y aunque se realiza las mejores medidas para su terapéutica aún falta mejor manejo de las mismas.

\section{Bibliografía}

Elsiever; Revista Española de Cirugía Ortopédica y Traumatología. (Junio de 2010). Fracturas abiertas. (J. M. Vivesa, P. C. Doussouxb, \& D. M. iGarinc, Productores) Recuperado el 14 de Diciembre de 2014, de Traumatologia y Ortopedia: http://apps.elsevier.es/watermark/ctl_servlet?_f=10\&pident_articulo=13184308\&pident_us uario=0\&pcontactid=\&pident_revista $=129 \&$ ty $=37 \&$ accion $=$ L\&origen=zonadelectura \&we b=zl.elsevier.es\&lan=es\&fichero=129v54n06a13184308pdf001.pdf

Gray, H. (1918). Anatomy of the human body. In H. Gray, Anatomy of the human body. Philadelfia: Lea \& Febiger. 


\section{Determinar las complicaciones de las fracturas expuestas de tibia en pacientes de 20-40 años en el hospital Teodoro Maldonado Carbo periodo 2014}

Vol. 3, núm. 3., (2019)

Maria Monserrate Ganchozo Pincay; Charles David Altamirano Olvera; Renato Paolo Patiño; Andrade; Javier Alexander Beltrán Bayas

Hospital Teodoro Maldonado Carbo. (2013). Reseña histórica. Recuperado el 12 de Sep de 2014, de http://www.htmc.gob.ec

Muller, M., Allgowe, M., \& Schneider. (1994). Manual de Osteosintesis. (S. -V. Iberica, Ed.) New York, USA: Científico Medico Barcelona.

Moore, K. A. (2007). Fundamentos de la Anatomia con orientacion clinica (2da edición ed.). Buenos Aires, Argentina: Editorial Medica Panemericana.

Muy Ilustre Municipalidad de Guayaquil. (2013). Alcaldía de guayaquil. Recuperado el 12 de Sep de 2014, de Geografía de la ciudad: http://www.guayaquil.gob.ec/la-ciudad/geografia

Revista Española de Cirugía Osteoarticular. (29 de junio de 2014). Indicaciones actuales de enclavado endomedular en las fracturas diafisarias del fémur y la tibia. Actualización. (D. E. Perelló, Productor) Recuperado el 14 de diciembre de 2014, de Traumatologia y Ortopedia: http://www.cirugia-

osteoarticular.org/adaptingsystem/intercambio/revistas/articulos/2347_Art58.pdf

Salminen, S. (2005). Tibials fractures in adults: Epidemiology, fracture patterns, nonunions, and fatigue fractures. University of Helsinki, Department of Pediatric Surgery. Helsinki: Helsinki University printing house.

Simon, R., \& Sherman, Scott. (2012). Emergencia en Ortopedia (Sexta Edición ed.). (D. C. AMOLCA, Trad.) USA, New York: AMOLCA.

Álvarez López, Alejandro, García Lorenzo, Yenima, Gutiérrez Blanco, Mario, \& Puentes Álvarez, Antonio. (2011). Fractura abierta de tibia: aspectos básicos. Revista Archivo Médico de Camagüey, 15(2), 388-399.

Gutiérrez Blanco, Mario, Álvarez López, Alejandro, \& García Lorenzo, Yenima. (2009). Tratamiento de las fracturas abiertas de tibia con pérdida de tejido óseo. Revista Archivo Médico de Camagüey, 13(4)

Testud, \& A., L. (1979). Tratado de Anatomia Humana (Vol. II). Barcelona, España: Salvat SA. 